Research Paper

\title{
Manual acupuncture relieves bile acid-induced itch in mice: the role of microglia and TNF- $\alpha$
}

\author{
Yu-Chen Lee, ${ }^{1,2 \#, ~ C h i a-H s i e n ~ L i n ~}{ }^{3 \#, ~ S h i h-Y a ~ H u n g ~} 4$, Hsin-Yi Chung², Sih-Ting Luo², Iona MacDonald ${ }^{2}$, \\ Yu-Ting Chu², Pei-Lin Lin ${ }^{5 凶}$, Yi-Hung Chen ${ }^{2,6,7 \varpi}$ \\ 1. Department of Acupuncture, China Medical University Hospital, Taichung 40402, Taiwan \\ 2. Graduate Institute of Acupuncture Science, China Medical University, Taichung 40402, Taiwan \\ 3. Department of Health Industry Management, Kainan University, No. 1 Kainan Road, Taoyuan 33857, Taiwan \\ 4. Department of Medical Laboratory Science and Biotechnology, China Medical University, Taichung 40402, Taiwan \\ 5. Department of Anesthesiology, National Taiwan University Hospital, Taipei 10002, Taiwan \\ 6. Chinese Medicine Research Center, China Medical University, Taichung 40402, Taiwan \\ 7. Department of Photonics and Communication Engineering, Asia University, Taiwan \\ \#: equal contribution \\ $\square$ Corresponding authors: pl15611@ntu.edu.tw (P.-L.L.); Tel: +886-2-23123456 (ext. 65518); yihungchen@mail.cmu.edu.tw (Y.-H.C.); Tel: +886-4-220-53366 (ext. \\ 3607)
}

(c) Ivyspring International Publisher. This is an open access article distributed under the terms of the Creative Commons Attribution (CC BY-NC) license (https://creativecommons.org/licenses/by-nc/4.0/). See http://ivyspring.com/terms for full terms and conditions.

Received: 2017.12.02; Accepted: 2018.05.27; Published: 2018.06.13

\begin{abstract}
Pruritus, or itch, is a frequent complaint amongst patients with cholestatic hepatobiliary disease and is difficult to manage, with many patients refractory to currently available antipruritic treatments. In this study, we examined whether manual acupuncture (MA) at particular acupoints represses deoxycholic acid (DCA)-induced scratching behavior and microglial activation and compared these effects with those induced by another pruritogen, 5'-guanidinonaltrindole (GNTI, a kappa opioid receptor antagonist). MA at Hegu (LI4) and Quchi (LII1) acupoints significantly attenuated DCA- and GNTI-induced scratching, whereas no such effects were observed at the bilateral Zusanli acupoints (ST36). Interestingly, GNTI-induced scratching was reduced similarly by both MA and electroacupuncture (EA) at the LI4 and LII 1 acupoints. MA at non-acupoints did not affect scratching behavior. Intraperitoneal injection of minocycline (a microglial inhibitor) reduced GNTI- and DCA-induced scratching behavior. In Western blot analysis, subcutaneous DCA injection to the back of the neck increased spinal cord expression of ionized calcium-binding adapter molecule 1 (lbal) and tumor necrosis factor-alpha (TNF- $\alpha$ ) as compared with saline injection, while MA at LI4 and LIII reduced these DCA-induced changes. Immunofluorescence confocal microcopy revealed that DCA-induced lbal-positive cells with thicker processes emanated from the enlarged cell bodies, while this effect was attenuated by pretreatment with MA. It is concluded that microglia and TNF- $\alpha$ play important roles in the itching sensation and MA reduces DCA-induced scratching behavior by alleviating spinal microglial activation. MA may be an effective treatment for cholestatic pruritus.
\end{abstract}

Key words: acupuncture, pruritogen, microglial activation, spinal cord, cholestatic pruritus

\section{Introduction}

Pruritus is an unpleasant sensation that elicits the desire or reflex to scratch [1] and is often associated with several disorders, such as atopic dermatitis [2], cholestasis [3], and uremic disease [4]. Pruritus is a troublesome symptom in patients with cholestatic liver disease, such as primary biliary cirrhosis, primary sclerosing cholangitis, intrahepatic cholestasis of pregnancy, and hepatitis C virus infection [5]. The pathogenesis of pruritus in cholestasis is uncertain, but some believe that bile acid accumulation may be the cause [6-8]. Levels of bile acids are elevated in serum and tissue during cholestasis and bile acids act as a pruritogen, which may diffuse from the systemic circulation into the skin [9]. Support for this theory comes from the fact that application of bile acids to the skin causes itch $[7,8]$ 
and one study has suggested that cholestatic pruritus is mediated by the bile acid-activated receptor, the G-protein-coupled receptor, TGR5, expressed by peptidergic neurons of mouse dorsal root ganglia and spinal cord [10]. It is well known that pruritus can be profoundly debilitating; orthotopic liver transplantation may be the only option for severe and intractable pruritus [11]. Further investigations are warranted into the causative mechanism and optimal treatment of cholestatic pruritus.

Microglia are central nervous system (CNS)-residing macrophages of erythromyeloid origin that are known to play immunoregulatory roles within the CNS [12]. In animal models of neuropathic pain in which a peripheral nerve is damaged, microglia become activated in the spinal dorsal horn. Morphologically, their cell bodies become hypertrophic with thickened and retracted processes, and cell numbers are increased [13]. Spinal microglia dramatically alter the expressions of many genes, including integrins, ionized calcium-binding adapter molecule-1 (Iba1), neurotransmitter receptors and intracellular kinases [14]. Activation of microglia releases a series of bioactive molecules, such as interleukin-1 $\beta$ (IL-1 $\beta)$, IL-6, tumor necrosis factor (TNF)- $\alpha$, proteases, brain-derived neurotrophic factor, and reactive oxygen species [15-17]. Recent research indicates that both compound $48 / 80$ and 5'-guanidinonaltrindole (GNTI) elicit pronounced scratching behavior and microglial activation in mouse spinal cord [18]. Previous studies have revealed the involvement of spinal microglia in pruritogen-induced itching, but their role in cholestatic pruritus is unclear.

Some studies have shown that acupuncture reduces histamine-induced itch in healthy volunteers $[19,20]$ and that both manual acupuncture (MA) and electroacupuncture (EA), but not pseudoacupuncture, reduce the maximal intensity of itch when applied to the site of histamine injection [21]. In our previous study, we found that EA at the Hegu (LI4) and Quchi (LI11), but not at the Zusanli acupoints (ST36), reduces pruritogen-induced scratching behavior in mice and microglial activation in mouse spinal cord [22]. In the present study, we examined whether MA (acupuncture without electrical stimulation) reduces deoxycholic acid (DCA)-induced scratching behavior and we compared the effects of MA with those of EA. We sought to define the relationship between MA and microglial activation in mouse spinal cord.

\section{Materials and methods}

\section{Animals}

We housed male ICR mice (25-30 g; BioLasco Taiwan Co., Ltd, Taiwan) in our animal facility under a 12:12 light/dark cycle with food and water available ad libitum for at least 4 days prior to the experiments, which were conducted between 10:00-18:00 h. China Medical University's Institutional Animal Care and Use Committee approved the experimental protocol, which was conducted according to guidance released by the Chinese Taipei Society of Laboratory Animal Sciences.

\section{Electroacupuncture (EA) and manual acupuncture (MA)}

EA procedures followed previously described experiments [23]. Mice were individually acclimated in rectangular observation boxes for $1 \mathrm{~h}$ before being anesthetized with $1.5 \%$ isoflurane. Under anesthesia, stainless steel acupuncture needles were inserted 2 $\mathrm{mm}$ deep at bilateral Zusanli points (ST-36), located at the proximal 1/5 site of the craniolateral surface of the leg, distal to the head of the tibia, in a depression between the muscles of the cranial tibia and the long digital extensor [24]. A non-acupoint was selected in the middle of the lateral deltoid muscle. In the itch study, a pair of stainless steel acupuncture needles was inserted $2 \mathrm{~mm}$ deep into the Hegu (LI4) and Quchi (LI11) acupoints. The murine LI4 is located on the dorsum of the hand, radial to the midpoint of the second metacarpal bone in the forefoot. The murine LI11 is located in the depression on the lateral end of the cubital crease, when the elbow is fully flexed [25]. EA stimuli were delivered by an EA Trio 300 stimulator (Ito, Japan) at an intensity of $2 \mathrm{~mA}$ for 20 min at $2 \mathrm{~Hz}$, using a $150 \mu$ s pulse width [26]. The MA procedures were the same as those in the EA protocol, without electrical stimulation.

\section{Pruritogen-evoked scratching behavior}

Mice were injected subcutaneously (s.c.) with saline, GNTI $(0.3 \mathrm{mg} / \mathrm{kg})$ or DCA $(8.3 \mathrm{mg} / \mathrm{kg})$ to the back of the neck. Scratching bouts were video recorded and counted manually for $40 \mathrm{~min}$, to minimize the effects of anesthesia [26, 27-31]. All test agents were administered in a dose volume of 10 $\mathrm{mL} / \mathrm{kg}$. Each bout involved a series of scratching behaviors: the stretching of the hind paw to the head, face, or back, rapid movement of the hind paw several times, then the moving of the paw to the floor.

\section{Western blot analysis of lbal and TNF- $\alpha$ levels}

Iba1 and TNF- $\alpha$ protein levels were determined by Western blot analysis, using the methods reported previously [32], with some modifications. Spinal cord segments from cervical C5 to C8 were extracted and cut into two halves (dorsal and ventral). Tissue obtained from the dorsal horn side was used for Western blot analysis. Tissue samples were homogenized in radioimmunoprecipitation assay 
buffer (RIPA Buffer) containing protease inhibitors and phosphatase inhibitors. Each sample of protein concentration was evaluated using a Pierce BCA Protein Assay Kit. Proteins $(30 \mu \mathrm{g})$ were resolved by $8-12 \%$ sodium dodecyl sulfate-polyacrylamide gel electrophoresis (SDS-PAGE) under reducing conditions and the gel was transferred to polyvinylidene difluoride (PVDF) membranes, then incubated overnight at $4^{\circ} \mathrm{C}$ with Iba1 (1:1000; ab5076; Abcam) antibody or TNF-a (1:1000; ab9739; Abcam) diluted in Tris-buffered saline (TBS) and probed with $\beta$-actin antibody (1:10000; GTX629630; GeneTex) as a standard. Membranes were incubated with secondary antibody (1:10,000; anti-rabbit IgG-HRP sc-2004; anti-mouse IgG-HRP sc-2005; or anti-goat IgG-HRP sc-2020; SantaCruz). Protein bands were detected and estimated.

\section{Analysis of lbal localization by confocal image analysis}

Immunofluorescence confocal microscopy was performed according to the method described by Lin et al. (2016, 2018), with some modifications [23, 33]. Tissue samples from C5-C8 spinal segments were blocked by PBS containing donkey serum $0.5 \%$ BSA and $0.4 \%$ Triton $\mathrm{X}-100$ before being treated with Iba1 (1:500, \#ab5076; Abcam) antibodies for 1 day. Sections were then incubated with $\mathrm{Cy}^{\mathrm{TM}} 3$ AffiniPure Donkey Anti-Goat IgG (H+L) (1:250, 705-165-003, Jackson ImmunoResearch Inc.) for $1 \mathrm{~h}$, mounted on slides with ProLong ${ }^{\mathrm{TM}}$ Gold antifade mountant (Thermo Fisher Scientific Inc.) and the coverslip was sealed with nail polish. Images were taken by a confocal microscope (model SP8 TCS; Leica, Heidelberg, Germany).

\section{Statistics}

Results are expressed as the means \pm standard error of the mean (S.E.M.). Statistical significance was analyzed using the Student's $t$-test or one-way analysis of variance (ANOVA), followed by a post hoc Dunnett's multiple comparison test to determine statistically significant between-group differences. $\mathrm{P}<$ 0.05 was considered significant.

\section{Results}

\section{Effects of MA or EA on GNTI-induced scratching behavior}

During the $40 \mathrm{~min}$ of assessment after s.c. GNTI administration $(0.3 \mathrm{mg} / \mathrm{kg})$, the mean number of scratching bouts was significantly reduced by both MA and EA at LI4 and LI11, compared with scratching in the control group, while MA at non-acupoints had no such effects (Fig. 1A). However, bilateral application of MA or EA at the ST-36 acupoints failed to reduce the mean number of scratches, compared with mice treated with GNTI alone (Fig. 1B).

A

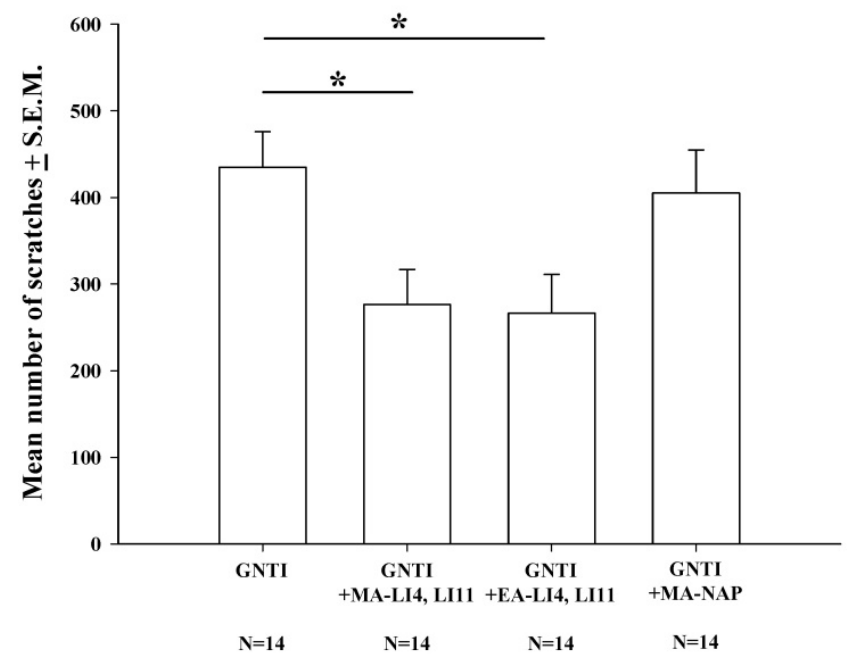

B

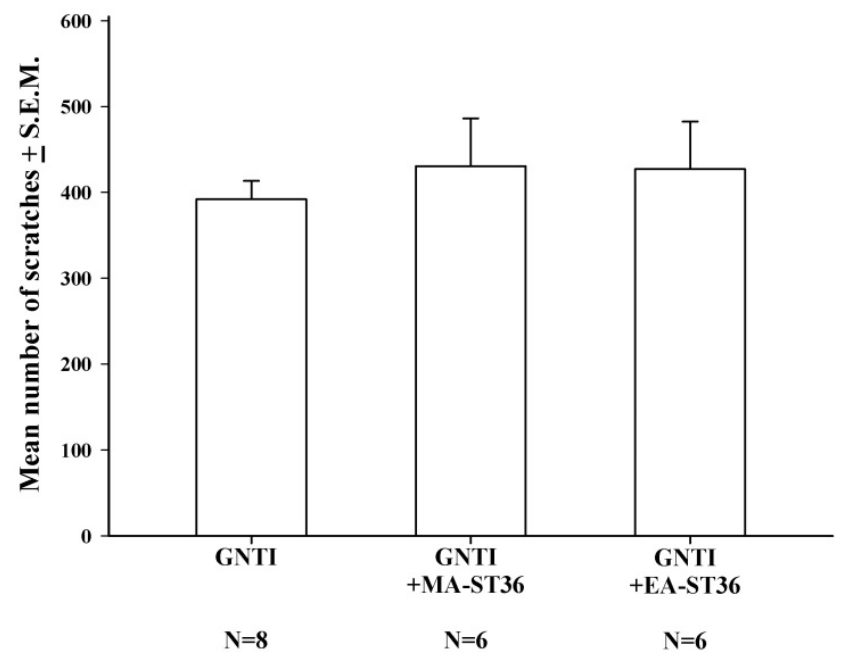

Figure 1. Effects of MA and EA on GNTI-induced scratching behavior. (A) MA or EA was applied to LI4 and LII1 acupoints 30 min before GNTI administration $(0.3 \mathrm{mg} / \mathrm{kg}, \mathrm{s} . \mathrm{c}$. $)$ to the back of the neck. The number of scratching bouts was counted for $40 \mathrm{~min}$ after GNTI injection. Both MA and EA at LI4 and LIII significantly attenuated GNTI-induced scratching behavior compared with scratching in the control group. Between-group comparisons for each group were performed by ANOVA, followed by the Dunnett's test ( ${ }^{*} \mathrm{P}<0.05$; the GNTI-only group served as the reference group). MA at non-acupoints had no effect upon scratching. (B) MA or EA was applied to bilateral ST36 acupoints 20 min before GNTI administration. The mean number of scratching bouts was counted for 40 min after GNTI injection. Neither MA nor EA at ST-36 reduced scratching behavior. NAP = Non-acupoint.

\section{Effects of MA on DCA-induced scratching behavior}

Compared with saline treatment, DCA (8.3 $\mathrm{mg} / \mathrm{kg}$ ) alone significantly increased the mean number scratches over the 40-min measurement period; this increase was significantly attenuated in mice treated with MA at LI4 and LI11 20 min prior to DCA administration, whereas MA at non-acupoints had no such effects (Fig. 2). 


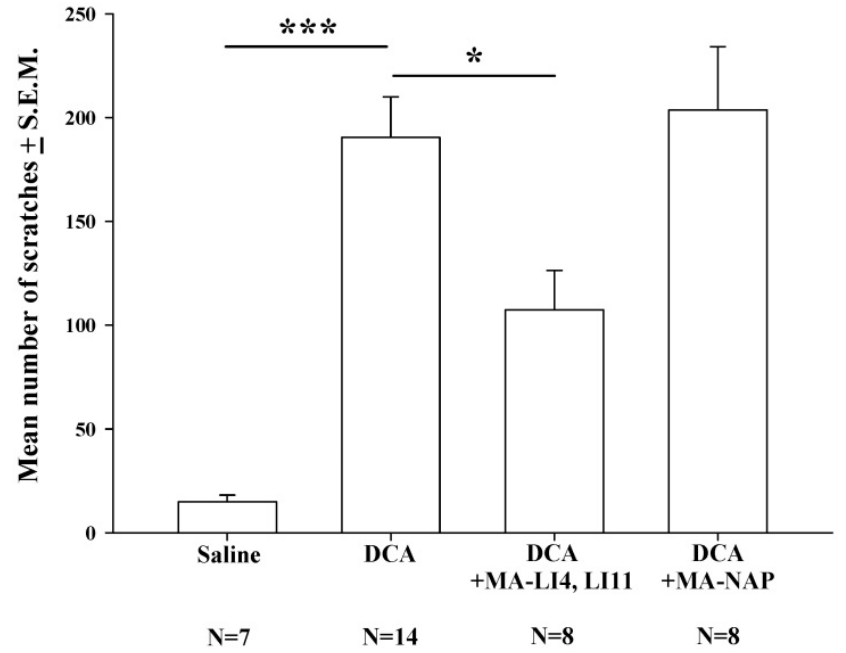

Figure 2. Effects of MA on DCA-induced scratching behavior. Mice were treated with saline, DCA $(8.3 \mathrm{mg} / \mathrm{kg})$ alone or in combination with MA to LI4 and LI1 1, or DCA plus MA at non-acupoints, and the number of scratching bouts was counted for $40 \mathrm{~min}$ in each group. DCA treatment increased scratching behavior compared with saline treatment. MA at LI4 and LI11 20 min prior to DCA administration attenuated DCA-induced scratching behavior. MA at non-acupoints had no effect upon scratching. Between-group comparisons for each group were performed by ANOVA, followed by the Dunnett's test (*P $<0.05$; *** $\mathrm{P}<0.001$; the DCA-only group served as the reference group). NAP = Non-acupoint.

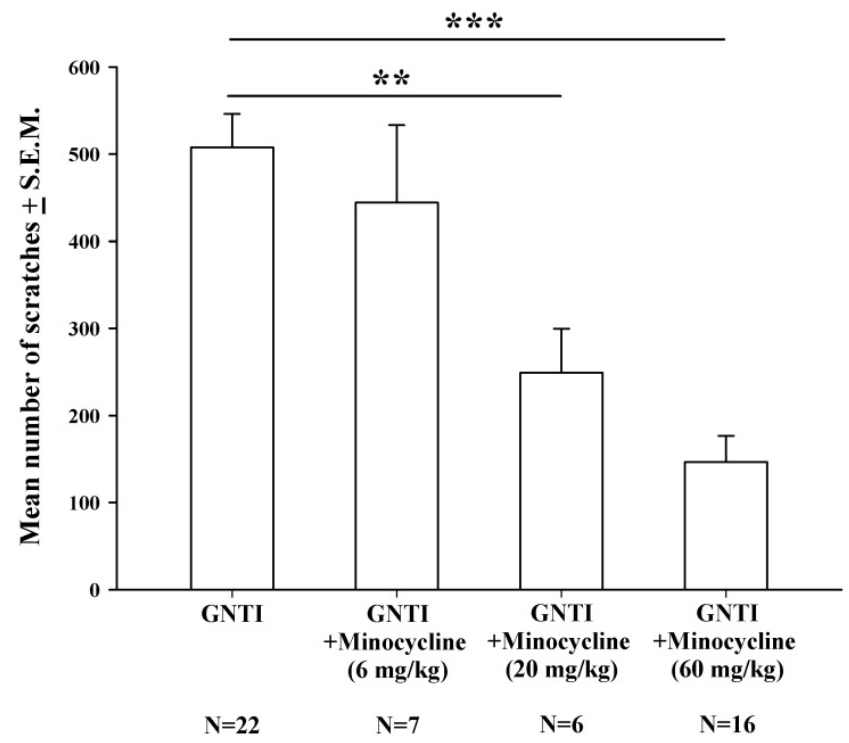

Figure 3. The effects of minocycline $(6-60 \mathrm{mg} / \mathrm{kg})$ upon GNTI-induced scratching behavior. Mice were pretreated with saline or minocycline $6 \mathrm{mg} / \mathrm{kg}, 20$ $\mathrm{mg} / \mathrm{kg}$, or $60 \mathrm{mg} / \mathrm{kg} 20 \mathrm{~min}$ before GNTI administration. The number of scratching bouts was counted for $40 \mathrm{~min}$ in each group. Minocycline $(20 \mathrm{mg} / \mathrm{kg}$ and $60 \mathrm{mg} / \mathrm{kg}$ ) dose-dependently reduced GNTI-induced scratching behavior. Between-group comparisons for each minocycline-treated group versus GNTI-treated animals were performed by ANOVA, followed by the Dunnett's test (**P $<0.01$; *** $\mathrm{P}<0.001$; the GNTI-only group served as the reference group).

\section{Effects of minocycline pretreatment upon GNTI-induced scratching behavior}

Minocycline is considered to be a specific inhibitor of microglial activation [34]. Intraperitoneal injection of minocycline at doses of $6 \mathrm{mg} / \mathrm{kg}, 20$ $\mathrm{mg} / \mathrm{kg}$, and $60 \mathrm{mg} / \mathrm{kg}$ was applied $20 \mathrm{~min}$ prior to GNTI administration. Minocycline $20 \mathrm{mg} / \mathrm{kg}$ and 60 $\mathrm{mg} / \mathrm{kg}$ dosages dose-dependently and significantly reduced the mean number of scratches induced by s.c. GNTI (0.3 mg/kg) (Fig. 3).

\section{Minocycline reduces GNTI-induced scratching behavior, with no further benefit from MA treatment}

Pretreatment with intraperitoneal (i.p.) minocycline $(20 \mathrm{mg} / \mathrm{kg}) 20 \mathrm{~min}$ prior to s.c. GNTI administration significantly reduced the numbers of scratching bouts compared with those associated with GNTI alone. The addition of MA treatment did not apparently further improve the reduction in scratching behavior in mice pretreated with minocycline (Fig. 4).

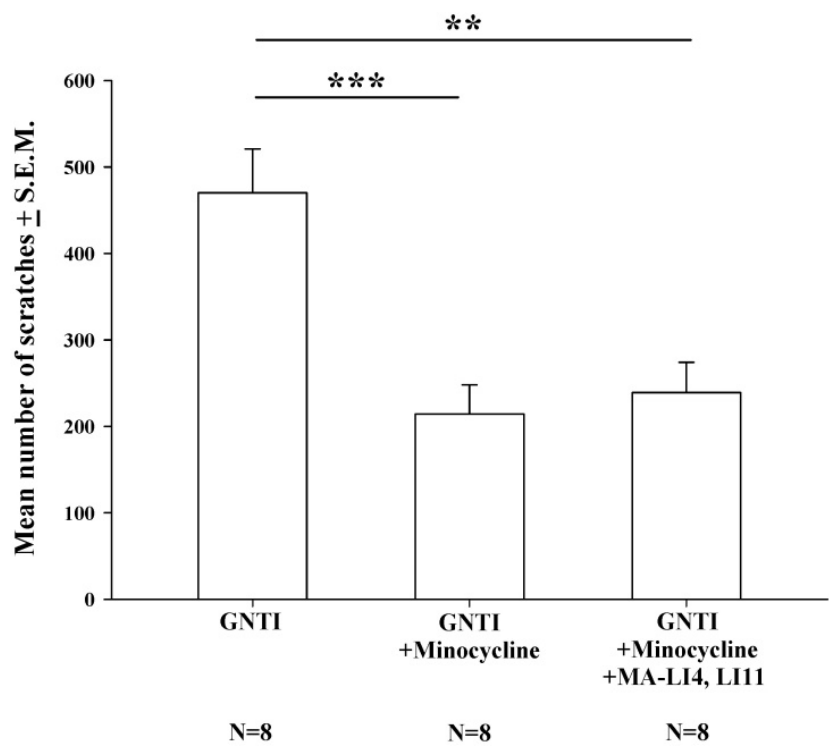

Figure 4. Minocycline alone reduces GNTI-induced scratching behavior, with no further reductions after the addition of MA. Mice were pretreated with minocycline $(20 \mathrm{mg} / \mathrm{kg})$ with or without MA at LI4 and LII 120 min before GNTI administration. Mice administered GNTI alone served as controls. The number of scratching bouts was counted for $40 \mathrm{~min}$ in each group. Scratching behavior was reduced by similar amounts in both minocycline-treated groups; MA did not further improve the reduction in GNTI-induced scratching behavior. Between-group comparisons for each group were performed by ANOVA, followed by the Dunnett's test ( $* * \mathrm{P}<0.01 ; * * * \mathrm{P}<0.001$; the GNTI-only group served as the reference group).

\section{Effects of minocycline pretreatment upon DCA-induced scratching behavior}

Intraperitoneal injections of minocycline at doses of $20 \mathrm{mg} / \mathrm{kg}$ and $60 \mathrm{mg} / \mathrm{kg}$ were administered $20 \mathrm{~min}$ prior to DCA administration $(8.3 \mathrm{mg} / \mathrm{kg})$. Compared with saline pretreatment, both pretreatment doses of minocycline $(20$ and $60 \mathrm{mg} / \mathrm{kg})$ resulted in significantly lower mean numbers of DCA-induced scratches (Fig. 5); this minocycline-induced reduction appeared to be dose-dependent.

MA pretreatment reduces DCA-induced lbal and TNF- $\alpha$ expression in dorsal horn spinal cord tissue

Western blotting was performed to verify that MA suppresses DCA-induced microglial activation. 
Spinal Iba1 and TNF- $\alpha$ levels were significantly increased after DCA injection, as shown in Fig. 6 \& 7 . In contrast, Iba1 and TNF- $\alpha$ levels in mice pretreated with MA closely matched respective levels in the saline group.

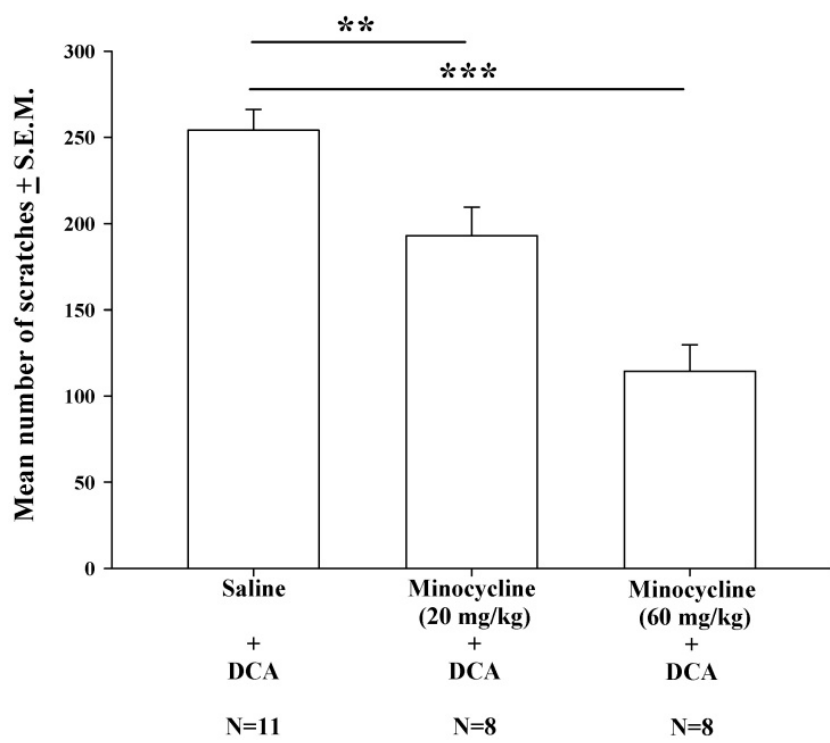

Figure 5. The effects of different doses of minocycline (20-60 mg/kg) upon DCA $(8.3 \mathrm{mg} / \mathrm{kg})$-induced scratching. Mice were pretreated with saline or minocycline $(20 \mathrm{mg} / \mathrm{kg}$ and $60 \mathrm{mg} / \mathrm{kg}) 20 \mathrm{~min}$ before DCA administration. The number of scratches was then counted for $40 \mathrm{~min}$ in each group. Minocycline reduced DCA-induced scratching behavior, in a dose-dependent manner. Between-group comparisons for each group were performed by ANOVA, followed by the Dunnett's test $(* * \mathrm{P}<0.01 ; * * * \mathrm{P}<0.001$; the Saline+DCA group served as the reference group).

\section{Saline DCA DCA+MA}
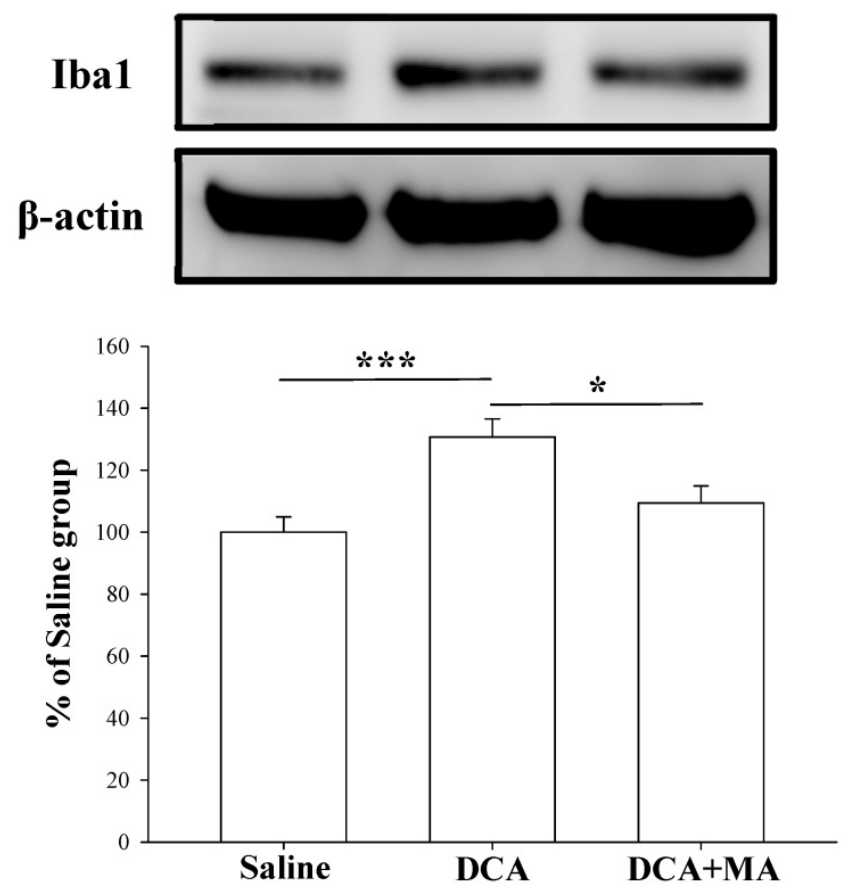

Figure 6. Pretreatment with MA reduced DCA-induced lbal expression in Western blot analysis. Spinal cord tissue from the dorsal horn (C5-C8) showed that DCA increased lbal expression compared with saline-treated tissue; pretreatment with MA reduced DCA-induced lbal expression. Between-group comparisons for each group were performed by ANOVA, followed by the Dunnett's test ( $* \mathrm{P}<0.05 ; * * * \mathrm{P}<0.001$; the DCA-only group served as the reference group).
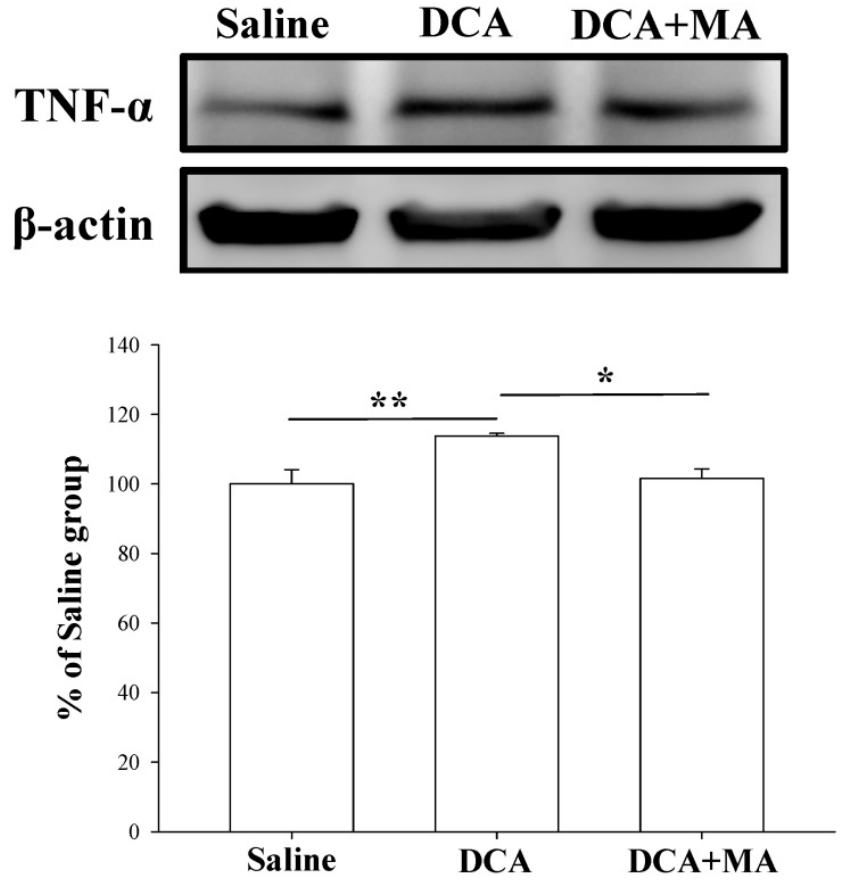

Figure 7. Pretreatment with MA reduced DCA-induced TNF- $\alpha$ expression in Western blot analysis. Spinal cord tissue from the dorsal horn dorsal horn (C5-C8) showed that DCA increased TNF- $\alpha$ expression compared with saline-treated tissue. Pretreatment with MA reduced DCA-induced TNF- $\alpha$ expression. Between-group comparisons for each group were performed by ANOVA, followed by the Dunnett's test ( $* \mathrm{P}<0.05$; **P $<0.01$; the DCA-only group served as the reference group).

\section{MA at LI4 and LII 1 reduced DCA-induced spinal microglia activation using immunofluorescence confocal microcopy}

The dorsal horn area was evaluated from cervical spinal cord sections. Iba1-stained tissue from saline-treated animals was characterized by small, weakly-stained cell bodies with several thin processes scattered throughout the gray and white matter (Fig. $8)$. In the DCA-treated animals, we found numerous strongly Iba $1^{+}$-labeled cells in both the gray and the white matter in the dorsal horn (Fig. 8); thick processes emanated from the enlarged cell bodies. Tissue from the DCA+MA (LI4, LI11) group had weakly-stained Iba1 ${ }^{+}$-labeled cell bodies with thinner processes compared to cells from the DCA group.

\section{Discussion}

We have previously reported that EA at LI4 and LI11 attenuates pruritogen-induced scratching behavior [22]. In the present study, we show that MA without electrical stimulation at LI4 and LI11 attenuated GNTI- and DCA-induced scratching behavior, as did i.p. injections of the microglial inhibitor, minocycline. In Western blot analysis, spinal Iba1 and TNF-a expression levels were increased in DCA-treated mice, but MA at LI4 and LI11 reversed TNF- $a$ expression. This is the first 


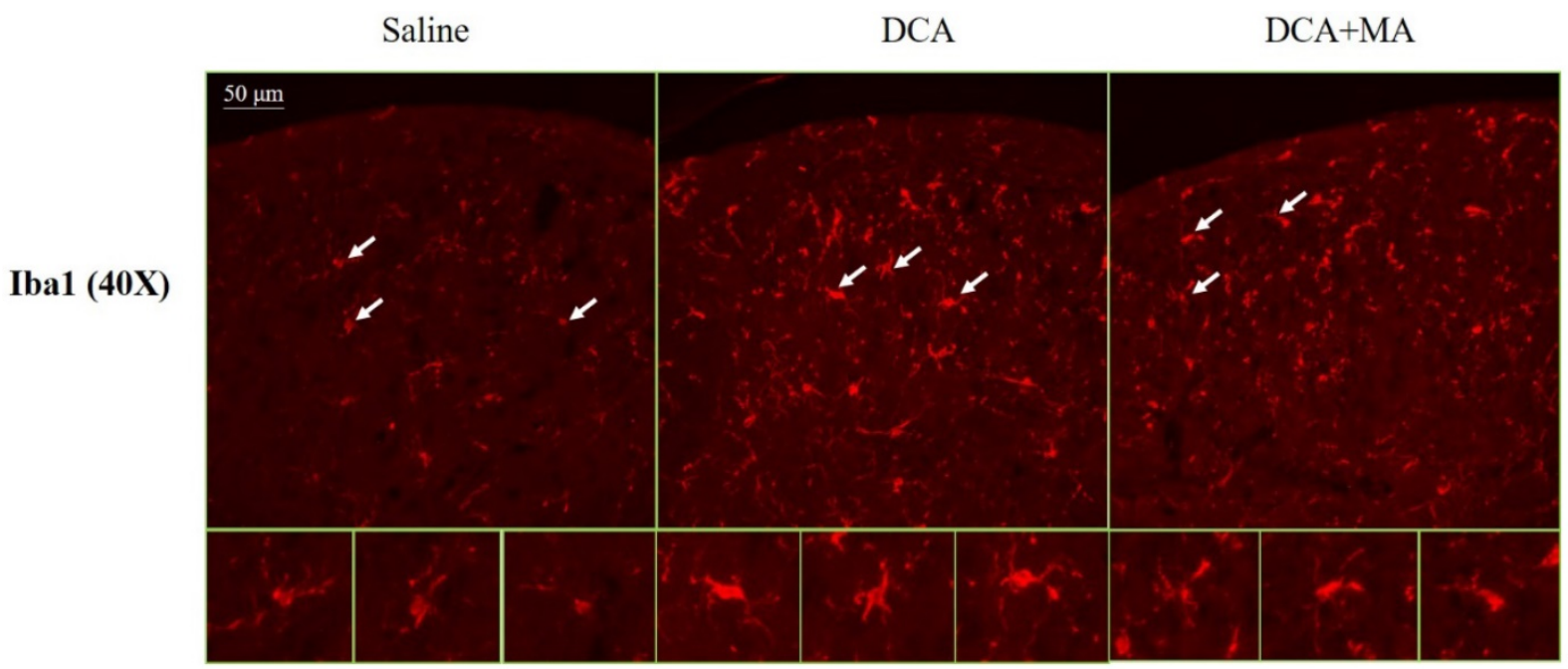

Figure 8. Ibal expression in the dorsal horn of spinal cord was assessed by immunofluorescence confocal microscopy. Representative immunofluorescent photomicrographs of Ibal expression in the cervical C5-C8 dorsal horn segments are shown from 3 groups; Saline, DCA, and DCA+MA. The lower panels depict enlarged Ibal images from their respective areas, as indicated by arrows.

report to show that MA treatment attenuates DCA-induced scratching behavior as well as spinal microglial and TNF- $\alpha$ activation, which suggests that acupuncture has an antipruritic effect in cholestatic pruritus.

Several clinical reports have described the effectiveness of acupuncture for the treatment of itch in humans $[19,21,35-38]$. Acupuncture stimulation is considered to be an effective antipruritic treatment if administered to dermatomes corresponding to affected sites or to adjacent dermatomes, supposedly due to kappa-opioid receptor activation [26]. Pharmacologic therapy is the mainstay of treatment for the management of cholestatic pruritus, according to recommendations issued by the American Association for the Study of Liver Disease and the European Association for the Study of the Liver. However, these medications are often limited by adverse effects and lack of therapeutic efficacy. Acupuncture may be an effective therapy for cholestatic pruritus. It is believed that $A \beta, A \delta$ and $C$ afferent fibers mediate MA signaling to the spinal cord [39]. According to the results of the present study, MA reduces the intensity of cholestatic pruritus with the same potency of EA.

Emerging evidence suggests that microglia are involved in itch processing. Zhang et al. [18] used GNTI and compound 48/80 (a mast cell activator) to induce itch and scratching in mice, and observed marked increases in spinal CD11b and phospho-p38 expression [18]. Further research has clarified that activated spinal cord microglia play a role in the development of 2,4-dinitrofluorobenzene (DNFB)induced pruritus using the same FKN/CX3CR1/ p38MAPK signaling pathways that participate in the development of neuropathic pain [40]. EA at LI4 and
LI11 reduced GNTI-induced expression of Iba1 and phospho-p38 in spinal cord [22]. Microglia are activated in the spinal cord under acute scratching induced by pruritogens (GNTI and compound 48/80) and DNFB-induced chronic itch in mice. Thus, levels of Iba1 and phospho-p38 expression can be regarded as markers of microglial activation.

Alemi et al. [10] detected TGR5 in peptidergic neurons of mouse dorsal root ganglion and spinal cord and they observed that the TGR5 receptor mediates bile acid-induced itch and analgesia. They propose the following mechanism for DCA-induced itch: bile acids activate TGR5 on sensory nerves, stimulating the release of neuropeptides in the spinal cord, which subsequently induce the release of gastrin-releasing peptide and opioids from spinal neurons [10]. The relationship between DCA-induced itch and microglia has not been clear. In our study, we investigated the potential role of microglia in a mouse model of cholestatic pruritus. Minocycline, an antibiotic of the tetracycline family, inhibits microglia in many paradigms and is one of the most commonly used agents for studying microglia involvement [34, 41]. We found that minocycline dose-dependently reduced GNTI- and DCA-induced scratching behavior. Minocycline alone reduced GNTI-induced scratching behavior; the addition of MA had no further effect. Our results strongly suggest that spinal microglia play a critical role in the pathogenesis of DCA-induced itching. Intriguingly, we found that MA alone at LI4 and LI11 is as effective as minocycline pretreatment at reducing scratching behavior. However, MA has fewer side effects than minocycline [42].

Several lines of evidence suggest that cytokines and chemokines are key mediators in itch [43]. A 
number of reports have indicated the involvement of the proinflammatory cytokine TNF- $\mathrm{a}$ in pain sensitization in the CNS [44]. TNF-a enhances the tetrodotoxin-resistant $\mathrm{Na}^{+}$current in DRG neurons $[45,46]$. It is known that TNF-a elicits spinal synaptic plasticity and inflammatory pain, partly via functional coupling with the transient receptor potential cation channel subfamily $\mathrm{V}$ member 1 (TRPV1) [47]. TNF- $\alpha$ modulates central sensitization via NMDA-induced currents [48]. Enhanced TNF- $\alpha$ expression is seen in the dorsal horn after spinal cord injury [49], while TNF-a in the spinal cord contributes to both chloroquine-induced acute itch and dry skin-induced chronic itch [50]. To evaluate whether TNF- $\alpha$ is involved in MA amelioration of bile acid-induced itching, we compared levels of TNF-a expression for pretreatment of MA at LI4 and LI11. In Western blot analysis, TNF-a expression in the spinal cord was upregulated after DCA injection and this effect was prevented by MA pretreatment at LI4 and LI11 acupoints. These results are consistent with the changes in Iba1 expression seen after DCA injection in mice pretreated with MA. Therefore, microglialderived TNF-a may play a key role in regulating bile acid-induced pruritus.

Immunofluorescence confocal microscopy revealed enlarged cell bodies and a greater number of cell processes in Iba1-positive cells in tissue from the DCA-treated group compared with Iba1-positive cells in tissue from the saline-treated group, whereas Iba1-positive cells from animals treated with DCA plus MA were smaller in size and cell processes were shorter than in cells from the DCA-only group. These results revealed that DCA induced microglial activation, while this effect was attenuated by pretreatment of MA. It is established that TNF- $\alpha$ immunoreactivity is increased after acute spinal cord injury and is localized in neurons, glial cells (including astrocytes, oligodendrocytes, and microglia), and endothelial cells in areas of the spinal cord adjacent to the lesion site [51]. In the present study, although we observed that DCA increased the expression of TNF- $\alpha$, we were unable to determine the source of TNF-a. Future research needs to address this issue, as it is important to determine whether the TNF-a is derived from microglia, astrocytes, neurons, or other cells.

In summary, this is the first report to indicate a beneficial effect of MA in DCA-induced itching. Accompanying reductions in microglial activation were similar to those observed with minocycline, which indicates that spinal microglia and TNF-a could provide leads for the development of novel therapeutic agents for the treatment of bile acid-induced itch. If MA proves to be an effective treatment for cholestatic pruritus, such therapy could avoid the side effects associated with pharmacologic agents and improve the treatment of itch.

\section{Acknowledgments}

This work was supported by grants MOST104-2320-B-039-020-MY2 and 106-2320-B-039019- from the Ministry of Science and Technology, Taipei, Taiwan and DMR-106-004 from China Medical University Hospital, Taiwan. This work was also financially supported by the "Chinese Medicine Research Center, China Medical University" from The Featured Areas Research Center Program within the framework of the Higher Education Sprout Project under the Ministry of Education (MOE) in Taiwan.

\section{Competing Interests}

The authors have declared that no competing interest exists.

\section{References}

Green D, Dong X. The cell biology of acute itch. J Cell Biol. 2016; 213: 155-161. Bieber T. Atopic dermatitis. N Engl J Med. 2008; 358: 1483-1494.

Kremer AE, Oude Elferink RP, Beuers U. Pathophysiology and current management of pruritus in liver disease. Clin Res Hepatol Gastroenterol. 2011; 35: 89-97.

4. Mettang T, Kremer AE. Uremic pruritus. Kidney Int. 2015; 87: 685-691.

5. Levy C. Management of pruritus in patients with cholestatic liver disease. Gastroenterol Hepatol (N Y). 2011; 7: 615-617.

6. Schoenfield LJ, Sjövall J, Perman E. Bile acids on the skin of patients with pruritic hepatobiliary disease. Nature. 1967; 213: 93-94.

7. Varadi DP. Pruritus induced by crude bile and purified bile acids. Experimental production of pruritus in human skin. Arch Dermatol. 1974; 109: 678-681.

8. Kirby J, Heaton KW, Burton JL. Pruritic effect of bile salts. Br Med J. 1974; 4: 693-695.

9. Lanzini A, De Tavonatti MG, Panarotto B, Scalia S, Mora A, Benini F, et al. Intestinal absorption of the bile acid analogue ${ }^{75} \mathrm{Se}$-homocholic acid-taurine is increased in primary biliary cirrhosis, and reverts to normal during ursodeoxycholic acid administration. Gut. 2003; 52: 1371-1375.

10. Alemi F, Kwon E, Poole DP, Lieu T, Lyo V, Cattaruzza F, et al. The TGR5 receptor mediates bile acid-induced itch and analgesia. J Clin Invest. 2013; 123: $1513-1530$.

11. Bhalerao A, Mannu S. Management of pruritus in chronic liver disease Dermatol Res Pract. 2015; 2015: 295891. DOI: 10.1155/2015/295891.

12. Gomez Perdiguero E, Klapproth K, Schulz C, Busch K, Azzoni E, Crozet L, et al. Tissue-resident macrophages originate from yolk-sac-derived erythro-myeloid progenitors. Nature. 2015; 518: 547-551.

13. Gu N, Peng J, Murugan M, Wang X, Eyo UB, Sun D, et al. Spinal microgliosis due to resident microglial proliferation is required for pain hypersensitivity after peripheral nerve injury. Cell Rep. 2016; 16: 605-614.

14. Tsuda M, Inoue K, Salter MW. Neuropathic pain and spinal microglia: a big problem from molecules in "small" glia. Trends Neurosci. 2005; 28: 101-107.

15. Milligan ED, Watkins LR. Pathological and protective roles of glia in chronic pain. Nat Rev Neurosci. 2009: 10: 23-36.

16. Kim D, You B, Jo EK, Han SK, Simon MI, Lee SJ. NADPH oxidase 2-derived reactive oxygen species in spinal cord microglia contribute to peripheral nerve injury-induced neuropathic pain. Proc Natl Acad Sci U S A. 2010; 107: 14851-14856.

17. Tsuda M, Beggs S, Salter MW, Inoue K. Microglia and intractable chronic pain. Glia. 2013; 61: 55-61.

18. Zhang Y, Dun SL, Chen YH, Luo JJ, Cowan A, Dun NJ. Scratching activates microglia in the mouse spinal cord. J Neurosci Res. 2015; 93: 466-474.

19. Belgrade MJ, Solomon LM, Lichter EA. Effect of acupuncture on experimentally induced itch. Acta Derm Venereol. 1984; 64: 129-133.

20. Pfab F, Hammes M, Bäcker M, Huss-Marp J, Athanasiadis GI, Tölle TR, et al. Preventive effect of acupuncture on histamine-induced itch: a blinded, randomized, placebo-controlled, crossover trial. J Allergy Clin Immunol. 2005; 116: 1386-1388.

21. Lundeberg T, Bondesson L, Thomas M. Effect of acupuncture on experimentally induced itch. Br J Dermatol. 1987; 117: 771-777. 
22. Lin JG, Lee YC, Tseng $\mathrm{CH}$, Chen DY, Shih CY, MacDonald I, et al. Electroacupuncture inhibits pruritogen-induced spinal microglial activation in mice. Brain Res. 2016; 1649: 23-29.

23. Chen YH, Yang HY, Lin CH, Dun NJ, Lin JG. Electroacupuncture attenuates 5 '-guanidinonaltrindole-evoked scratching and spinal c-Fos expression in the mouse. Evid Based Complement Alternat Med. 2013; 2013: 319124. DOI: $10.1155 / 2013 / 319124$

24. He TF, Yang WJ, Zhang SH, Zhang CY, Li LB, Chen YF. Electroacupuncture inhibits inflammation reaction by upregulating vasoactive intestinal peptide in rats with adjuvant-induced arthritis. Evid Based Complement Alternat Med. 2011; 2011: 290489. DOI: 10.1155/2011/290489.

25. World Health Organization. WHO standard acupuncture point locations in the Western Pacific region. Manila, Philippines: World Health Organization Western Pacific Region Press; 2008.

26. Han JB, Kim CW, Sun B, Kim SK, Lee MG, Park DS, et al. The antipruritic effect of acupuncture on serotonin-evoked itch in rats. Acupuncture and Electrotherapy Research. 2008; 33: 145-156.

27. Enerbäck L. Mast cells in rat gastrointestinal mucosa. 2. Dye-binding and metachromatic properties. Acta Pathol Microbiol Scand. 1966; 66: 303-312.

28. Sugimoto $\mathrm{Y}$, Umakoshi K, Nojiri N, Kamei C. Effects of histamine $\mathrm{H}_{1}$ receptor antagonists on compound 48/80-induced scratching behavior in mice. Eur J Pharmacol. 1998; 351: 1-5.

29. Nojima H, Simons CT, Cuellar JM, Carstens MI, Moore JA, Carstens E. Opioid modulation of scratching and spinal c-fos expression evoked by intradermal serotonin. J Neurosci. 2003; 23: 10784-10790.

30. Inan S, Dun NJ, Cowan A. Nalfurafine prevents 5'-guanidinonaltrindole- and compound 48/80-induced spinal c-fos expression and attenuates 5 '-guanidinonaltrindole-elicited scratching behavior in mice. Neuroscience. 2009; 163: 23-33.

31. Yamamoto A, Kuyama S, Kamei C, Sugimoto Y. Characterization of scratching behavior induced by intradermal administration of morphine and fentanyl in mice. Eur J Pharmacol. 2010; 627: 162-166.

32. Hung WT, Hong X, Christenson LK, McGinnis LK. Extracellular vesicles from bovine follicular fluid support cumulus expansion. Biol Reprod. 2015; 93: 117. DOI: 10.1095/biolreprod.115.132977.

33. Lin JG, Lee YC, Tu CH, Iona MacDonald, Chung HY, Luo ST, Hung SY, Chen HY. Histamine H1 Receptor Antagonists Facilitate Electroacupuncture Analgesia. Am J Chin Med, 2018; 46:55-68.

34. Tikka T, Fiebch BL. Minocycline, a tetracycline derivative, is neuroprotective against excitotoxicity by inhibiting activation and proliferation of microglia. J Neurosci. 2001, 21:2580-2588.

35. Chou CY, Cheng YW, Kao MT, Huang CC. Acupuncture in haemodialysis patients at the Quchi (LI11) acupoint for refractory uraemic pruritus. Nephrol Dial Transplant. 2005; 20: 1912-1915.

36. Pfab F, Huss-Marp J, Gatti A, Fuqin J, Athanasiadis GI, Irnich D, et al. Influence of acupuncture on type I hypersensitivity itch and the wheal and flare response in adults with atopic eczema - a blinded, randomized, placebo-controlled, crossover trial. Allergy. 2010; 65: 903-910.

37. Pfab F, Kirchner MT, Huss-Marp J, Schuster T, Schalock PC, Fuqin J, et al. Acupuncture compared with oral antihistamine for type I hypersensitivity itch and skin response in adults with atopic dermatitis: a patient- and examiner-blinded, randomized, placebo-controlled, crossover trial. Allergy. 2012; 67: 566-573

38. Stellon A. The use of laser acupuncture for the treatment of neurogenic pruritus in a child - a case history. Acupunct Med. 2005; 23: 31-33.

39. Zhao ZQ. Neural mechanism underlying acupuncture analgesia. Prog Neurobiol. 2008; 85: 355-75.

40. Zhang Y, Yan J, Hu R, Sun Y, Ma Y, Chen Z, et al. Microglia are involved in pruritus induced by DNFB via the CX3CR1/p38 MAPK pathway. Cell Physiol Biochem. 2015; 35: 1023-1033.

41. Möller T, Bard F, Bhattacharya A, Biber K, Campbell B, Dale E, et al. Critical data-based re-evaluation of minocycline as a putative specific microglia inhibitor. Glia. 2016; 64: 1788-1794.

42. Witt CM, Pach D, Brinkhaus B, Wruck K, Tag B, Mank S, et al. Safety of acupuncture: results of a prospective observational study with 229,230 patients and introduction of a medical information and consent form. Forsch Komplementmed. 2009; 16: 91-97.

43. Storan ER, O'Gorman SM, McDonald ID, Steinhoff M. Role of cytokines and chemokines in itch. Handb Exp Pharmacol. 2015; 226: 163-176.

44. Leung L, Cahill CM. TNF- $\alpha$ and neuropathic pain - a review. J Neuroinflammation. 2010; 7: 27. DOI: 10.1186/1742-2094-7-27.

45. Jin, X, Gereau RW 4th. Acute p38-mediated modulation of tetrodotoxin-resistant sodium channels in mouse sensory neurons by tumor necrosis factor- $a$. J Neurosci. 2006; 26: 246-255.

46. Czeschik JC, Hagenacker T, Schäfers M, Büsselberg D. TNF-a differentially modulates ion channels of nociceptive neurons. Neurosci Lett. 2008; 434: 293-298.

47. Park CK, Lü N, Xu ZZ, Liu T, Serhan CN, Ji RR. Resolving TRPV1- and TNF-a-mediated spinal cord synaptic plasticity and inflammatory pain with neuroprotectin D1. J Neurosci. 2011; 31: 15072-15085.

48. Zhang L, Berta T, Xu ZZ, Liu T, Park JY, Ji RR. TNF- $a$ contributes to spinal cord synaptic plasticity and inflammatory pain: distinct role of TNF receptor subtypes 1 and 2. Pain. 2011; 152: 419-427.
49. Peng XM, Zhou ZG, Glorioso JC, Fink DJ, Mata M. Tumor necrosis factor- $a$ contributes to below-level neuropathic pain after spinal cord injury. Ann Neurol. 2006; 59: 843-851.

50. Miao X, Huang Y, Liu TT, Guo R, Wang B, Wang XL, et al. TNF-a/TNFR1 signaling is required for the full expression of acute and chronic itch in mice via peripheral and central mechanisms. Neurosci Bull. 2017; DOI: 10.1007/s12264-017-0124-3.

51. Yan P, Li Q, Kim GM, Xu J, Hsu CY, Xu XM. Cellular localization of tumor necrosis factor- $\alpha$ following acute spinal cord injury in adult rats. J Neurotrauma. 2001; 18: 563-568. 